\title{
Recurrent ectopic pregnancy as a unique clinical sub group: a case control study
}

\author{
Alice Hurrell, Oliver Reeba ${ }^{*}$ and Odejinmi Funlayo
}

\begin{abstract}
Background: Women with recurrent ectopic pregnancy (EP) represent a unique cohort of patients in whom diagnostic expertise is paramount. We determined whether recurrent EP is associated with significant differences in patient demographics, clinical presentation, risk factors and surgical findings when compared with primary EP.

Methods: A retrospective case-control study of all EPs diagnosed from 2003 to 2014, at Whipps Cross University Hospital, London.

Results: In the above period 849 EPs were surgically managed (758 primary EPs and 91 recurrent EPs). Recurrent EPs were significantly older than primary EPs ( $32.2 \pm 5.08$ vs. $30.5 \pm 5.83$ years, $p<0.05)$. They presented at a significantly earlier gestation ( $5.99 \pm 1.08$ vs. $6.52 \pm 1.81$ weeks, $p<0.05$ ) and with a significantly lower primary $\beta H C G$ (3176 \pm 7350 vs. $6243 \pm 12,282, p<0.05)$. Recurrent EPs were significantly more likely to have a positive history of tubal or pelvic surgery ( $61.5 \%$ vs. $3.5 \%, p<0.05$ and 53.8 vs. $14 \%, p<0.05)$. At surgery, primary EPs had a significantly greater volume of hemoperitoneum (592 \pm 850 vs. $249 \pm 391 \mathrm{ml}, \mathrm{p}<0.05)$, whereas recurrent EPs were significantly more likely to have contralateral pathology ( $31.1 \mathrm{vs.} 9.8 \%, p<0.05$ ). Regression analysis showed that the parameters of age, gestational age at presentation, first $\beta H C G$ level, positive history of previous tubal surgery and previous ectopic pregnancy differ in women at risk of a recurrent EP when compared to women not at risk of a recurrent ectopic (AUC, 0.844).
\end{abstract}

Conclusions: We conclude that recurrent EPs may represent a unique sub-group of patients with EP.

Keywords: Ectopic pregnancy, Diagnosis, Recurrent ectopic pregnancy, Laparoscopy

\section{Background}

An ectopic pregnancy is any pregnancy that implants outside the uterine cavity. The vast majority of ectopic pregnancies implant in the fallopian tube. Despite efforts at primary prevention, the incidence of EP has been stable over recent years, at 11.1 per 1000 pregnancies (The Management of Tubal Pregnancy 2004) though there has been a significant improvement in the mortality from EP as demonstrated in the last UK triennial report (Saving Mothers' Lives 2006). Creanga et al. estimated trends in EP mortality and examined characteristics of recently hospitalized women who died as a result of EP in the United States and found that the EP mortality ratio declined by $56.6 \%$, from 1.15 to 0.50 deaths per 100,000

*Correspondence: reeba.oliver@gmail.com

Department of Gynaecology, Whipps Cross University Hospital, Barts

Health Trust, Whipps Cross Road, Leytonstone, London E11 1NR, UK live births between 1980-1984 and 2003-2007 (Creanga et al. 2011).

Despite the declining mortality ratio, being diagnosed with an EP proves an anxiety-provoking time for any woman. In addition to the loss of the pregnancy, there may be acute and chronic implications for both general and reproductive health. All identified risk factors in the many multiple studies are to date maternal: Chlamydia trachomatis infection, pelvic inflammatory disease, smoking, tubal surgery, assisted reproductive techniques, previous miscarriages, previous dilatation and curettage and endometriosis. The risk of recurrent EP is greatly increased compared to that of primary EP and reported incidence ranges from 6 to $18 \%$, with a trend towards higher rates of recurrence after salpingotomy (Jurkovic and Wilkinson 2011; Ankum et al. 1996; Schoen and Nowak 1975). In spite of its identification as being

\section{望 Springer}

(c) 2016 Hurrell et al. This article is distributed under the terms of the Creative Commons Attribution 4.0 International License (http://creativecommons.org/licenses/by/4.0/), which permits unrestricted use, distribution, and reproduction in any medium, provided you give appropriate credit to the original author(s) and the source, provide a link to the Creative Commons license, and indicate if changes were made. 
contributory to risk the incidence and occurrence of recurrent EP has not been assessed in research. The challenge of secondary prevention is related to a paucity of modifiable risk factors.

The purpose of our study is to determine whether there is a significant difference in patient demographics, clinical presentation, risk factors and surgical findings between women presenting with primary or recurrent EP. If present, this may help to pre operatively identify women likely to have a recurrent EP.

\section{Objectives}

To determine whether recurrent EP is associated with any significant differences in patient demographics, clinical presentation, risk factors and surgical findings when compared to primary EP.

\section{Methods}

\section{Study design}

A retrospective case-control study of all EPs diagnosed from January 2003 to July 2014, at Whipps Cross University Hospital, London. Data for each surgically managed case of EP presenting to the hospital was entered prospectively into a dedicated database. Exclusion criteria included: non-surgically managed EP and incomplete dataset.

Patient details were collated and variables were collected as below in Table 1 .

\section{Statistical analysis}

Data was collected and stored on an Excel spreadsheet. Caldicott guidelines were strictly adhering to in the collection and storage of personal information (Crook 2003). Results were analyzed and compared using the Student's $t$ test and Fisher's test. Parametric tests were used, as all data passed the test of normality. Student's t test was used for numerical data and Fisher's exact test for categorical data. Significance was set at a p value of $<0.05$. Multivariate logistic regression analysis was performed using the significant variables from the univariate analysis. Analysis was performed using Graphpad Prism, Version 6.0 (Graphpad software, San Diego, USA).

\section{Results}

There were 849 consecutive EPs diagnosed and managed surgically from January 2003 to July 2014. Of these, 758 were primary EPs and 91 were recurrent EPs. Of these 52, $6.2 \%$ of the study cohort was not tubal EPs: 29 cornual and one repeat cornual EP, 19 ovarian with no recurrent EPs. There were 1 peritoneal, 2 CS scar ectopics, 1 heterotropic and 1 rudimentary horn pregnancy. Non tubal and tubal EPs were analysed together.
Women with recurrent EPs were significantly older than women with primary EPs $(32.2 \pm 5.08$ vs. $30.5 \pm 5.83$ years, $\mathrm{p}<0.05)$. They presented at a significantly earlier gestation $(5.99 \pm 1.08$ vs. $6.52 \pm 1.81$ weeks gestation, $\mathrm{p}<0.05$ ) and with a significantly lower primary $\beta$ HCG (3176 \pm 7350 vs. $6243 \pm 12,282$ IU/l, p < 0.05) (Table 2).

There was no significant difference in symptoms at the time of clinical presentation between women with primary or recurrent EP. However, there was a non-significant trend towards a greater proportion of patients with primary EP presenting with shoulder tip pain (10.5\% of primary EPs vs. $4.4 \%$ of recurrent EPs, $p=0.089$ ) (Table 2). This is presumably in keeping with women with primary EPs presenting with a significantly greater volume of hemoperitoneum (see Table 3). Women with recurrent EPs had a significantly lower primary $\beta$ HCG, in association with the significantly earlier gestation at time of clinical presentation (Table 2). Women with recurrent EP were also significantly more likely to have a transvaginal ultrasound scan.

Women with recurrent EP were unsurprisingly significantly more likely to have had previous tubal surgery or previous pelvic surgery (Table 2). However, there was otherwise no significant difference in risk factors between women presenting with recurrent or primary EP. It is noteworthy that women with recurrent EP were not significantly more likely to have had previous pelvic inflammatory disease (Table 2).

There were no significant differences in scan findings between the two sub-groups (Table 2). At the time of surgery, primary EP was significantly associated with greater volume of hemoperitoneum ( $592 \pm 850$ vs. $249 \pm 391 \mathrm{ml}$, $\mathrm{p}<0.05)$. Women with recurrent EP were significantly more likely to have contralateral pathology (31.1 vs. $9.8 \%, \mathrm{p}<0.05)$ and there was a non-significant trend towards a greater proportion of peri-hepatic adhesions ( 13.5 vs. $7.6 \%, p=0.066)$. In association with the desire to preserve future fertility, women with recurrent EP were significantly less likely to have a salpingectomy (57.1 vs. $83.3 \%, \mathrm{p}<0.05)$ and significantly more likely to have a salpingotomy (37.4 vs. $10.9 \%, \mathrm{p}<0.05$ ) (see Table 3 ).

The significant preoperative factors from the univariate analysis were entered into a logistic regression analysis. The predicted probabilities from the same were used to construct a ROC curve (Fig. 1). The AUC calculated was 0.844 , which indicates good differentiation between the two groups. Thus the parameters of age, gestational age at presentation, level of first $\beta$ HCG, positive history of previous tubal surgery and previous EP differ in women at risk of a recurrent EP when compared to women not at risk of a recurrent ectopic (Table 4). 
Table 1 Patient details and variables

\begin{tabular}{|c|c|c|c|c|}
\hline Variable & Type of variable & $\begin{array}{l}\text { Primary } \\
{[\mathrm{P}] / \text { secondary }[\mathrm{S}]}\end{array}$ & Description & $\begin{array}{l}\text { Units of measurement } \\
\text { [P present, } A \text { absent }]\end{array}$ \\
\hline Age & Demographic characteristic & $P$ & Age of each patient & Years \\
\hline Parity & Demographic characteristic & $P$ & No of deliveries & Number \\
\hline Gestation & Demographic characteristic & $P$ & Number of weeks of pregnancy & Weeks \\
\hline Pain & Clinical characteristic & $P$ & Presenting with abdominal pain & $\mathrm{P} / \mathrm{A}$ \\
\hline Vomiting & Clinical characteristic & $P$ & $\begin{array}{l}\text { In association with the other symp- } \\
\text { toms }\end{array}$ & $\mathrm{P} / \mathrm{A}$ \\
\hline Bleeding & Clinical characteristic & P & Vaginal bleeding & $\mathrm{P} / \mathrm{A}$ \\
\hline Diarrhoea & Clinical characteristic & $P$ & $\begin{array}{l}\text { In association with the other symp- } \\
\text { toms }\end{array}$ & $\mathrm{P} / \mathrm{A}$ \\
\hline Shoulder tip pain & Clinical characteristic & $P$ & $\begin{array}{l}\text { in association with the other symp- } \\
\text { toms }\end{array}$ & $\mathrm{P} / \mathrm{A}$ \\
\hline Syncope & Clinical characteristic & $P$ & $\begin{array}{l}\text { In association with the other symp- } \\
\text { toms }\end{array}$ & $\mathrm{P} / \mathrm{A}$ \\
\hline Smoking & Risk factor & $P$ & Known long term smoking & $\mathrm{P} / \mathrm{A}$ \\
\hline Previous tubal surgery & Risk factor & P & Due to previous tubal pathology & $\mathrm{P} / \mathrm{A}$ \\
\hline Previous pelvic surgery & Risk factor & $P$ & Due to previous pelvic pathology & $\mathrm{P} / \mathrm{A}$ \\
\hline Previous miscarriage & Risk factor & $P$ & $\begin{array}{l}\text { Diagnosed as miscarriage by scan } \\
\text { and histopathology }\end{array}$ & $\mathrm{P} / \mathrm{A}$ \\
\hline $\begin{array}{l}\text { Previous termination of } \\
\text { pregnancy }\end{array}$ & Risk factor & $P$ & $\begin{array}{l}\text { Diagnosed as intra uterine pregnancy } \\
\text { by scan and histopathology }\end{array}$ & $\mathrm{P} / \mathrm{A}$ \\
\hline Assisted reproduction & Risk factor & $P$ & Previous pathology & $\mathrm{P} / \mathrm{A}$ \\
\hline Previous infertility & Risk factor & $P$ & Previous pathology & $\mathrm{P} / \mathrm{A}$ \\
\hline Pelvic inflammatory disease & Risk factor & $\mathrm{P}$ & Previous pathology & $\mathrm{P} / \mathrm{A}$ \\
\hline $\begin{array}{l}\text { Use of intrauterine contra- } \\
\text { ceptive device }\end{array}$ & Risk factor & $P$ & As contraceptive & $\mathrm{P} / \mathrm{A}$ \\
\hline Initial beta HCG level & Investigation & S & Blood test & $\mathrm{mlU} / \mathrm{ml}$ \\
\hline $\begin{array}{l}\text { Transvaginal/trans-abdomi- } \\
\text { nal scan }\end{array}$ & Investigation & S & Ultrasound scan & $\begin{array}{l}\text { Performed/ } \\
\text { not performed }\end{array}$ \\
\hline Adnexal mass & Investigation & S & Scan finding & $\mathrm{P} / \mathrm{A}$ \\
\hline Gestational sac & Investigation & S & Scan finding & $\mathrm{P} / \mathrm{A}$ \\
\hline Fetal heartbeat & Investigation & S & Scan finding & $\mathrm{P} / \mathrm{A}$ \\
\hline Fluid in Pouch of Douglas & Investigation & S & Scan finding & $\mathrm{P} / \mathrm{A}$ \\
\hline Empty uterus & Investigation & S & Scan finding & $\mathrm{P} / \mathrm{A}$ \\
\hline Hemoperitoneum & Investigation & $S$ & Scan findings & $\mathrm{P} / \mathrm{A}$ \\
\hline Laparotomy & Treatment & S & Intra operative findings & $\mathrm{P} / \mathrm{A}$ \\
\hline Tubal ectopic pregnancy & Treatment & S & Intra operative findings & $\mathrm{P} / \mathrm{A}$ \\
\hline Salpingectomy & Treatment & S & Intra operative findings & $\mathrm{P} / \mathrm{A}$ \\
\hline Salpingotomy & Treatment & S & Intra operative findings & $\mathrm{P} / \mathrm{A}$ \\
\hline Hemoperitoneum & Treatment & S & Intra operative findings & $\mathrm{P} / \mathrm{A}$ \\
\hline $\begin{array}{l}\text { Presence of hepatic adhe- } \\
\text { sions }\end{array}$ & Treatment & S & Intra operative findings & $\mathrm{P} / \mathrm{A}$ \\
\hline Contralateral pathology & Treatment & S & Intra operative findings & $\mathrm{P} / \mathrm{A}$ \\
\hline
\end{tabular}

\section{Discussion}

Our study shows that women with recurrent EP may represent a distinct subgroup of patients with EP. This is particularly in relation to awareness of the condition and early presentation, as they are significantly more likely to present at an earlier gestation, with a lower BHCG and significantly less hemoperitoneum. Universally, women treated for EP are informed of the approximately $10 \%$ risk of recurrence of EP and they are advised to have an early pregnancy ultrasound scan in any future pregnancy. Our results contrast with a previous study of recurrent $E P$, which found a non-significant trend towards a higher $\beta$ HCG (Butts et al. 2003). These contrasting results may represent our thorough post-operative counseling and 
Table 2 The comparison of the baseline characteristics

\begin{tabular}{|c|c|c|c|c|c|}
\hline & \multirow{2}{*}{$\begin{array}{l}\text { Recurrent ectopic } \\
\text { Mean }( \pm S D) \text { or \% (n) }\end{array}$} & \multicolumn{4}{|l|}{ Primary ectopic } \\
\hline & & Mean $( \pm S D)$ or \% (n) & $\mathrm{p}$ value & $\begin{array}{l}\text { Mean difference } \\
\text { or odds ratio }\end{array}$ & $95 \% \mathrm{Cl}$ \\
\hline \multicolumn{6}{|c|}{ Baseline characteristics of women with EP } \\
\hline $\mathrm{Age}^{\mathrm{a}}$ & $32.2(5.08)$ & $30.5(5.83)$ & 0.0049 & -1.65 & -2.78 to -0.51 \\
\hline \multicolumn{6}{|l|}{ Parity $1^{\mathrm{b}}$} \\
\hline PO & $37.5(33 / 88)$ & $44.7(327 / 732)$ & 0.2127 & 0.7431 & 0.471 to 1.172 \\
\hline P1-P3 & $54.5(48 / 88)$ & $51.8(379 / 732)$ & 0.6526 & 1.118 & 0.717 to 1.742 \\
\hline P4 or more & $8.0(7 / 88)$ & $3.6(26 / 732)$ & 0.0755 & 2.347 & 0.9872 to 5.578 \\
\hline Gestation $^{\mathrm{a}}$ & $5.99(1.08)$ & $6.52(1.81)$ & 0.0001 & 0.5402 & 0.2728 to 0.8076 \\
\hline \multicolumn{6}{|c|}{ Risk factors for EP in women with primary and recurrent EP } \\
\hline Smoking & $18.7(17 / 91)$ & $14.6(108 / 741)$ & 0.3498 & 1.346 & 0.7650 to 2.370 \\
\hline Previous tubal surgery & $61.4(54 / 88)$ & $3.5(26 / 740)$ & $<0.0001$ & 43.62 & 24.40 to 77.97 \\
\hline Previous pelvic surgery & $53.8(49 / 91)$ & $14.0(104 / 743)$ & $<0.0001$ & 7.168 & 4.519 to 11.37 \\
\hline Previous miscarriage & $27.5(25 / 91)$ & $24.1(179 / 742)$ & 0.5185 & 1.191 & 0.7297 to 1.945 \\
\hline Previous TOP & $14.3(13 / 91)$ & $18.7(139 / 743)$ & 0.3875 & 0.7242 & 0.3913 to 1.340 \\
\hline Assisted reproduction & $3.3(3 / 91)$ & $4.3(32 / 743)$ & 1.0 & 0.7575 & 0.2272 to 2.526 \\
\hline Previous infertility & $12.1(11 / 91)$ & $7.8(58 / 743)$ & 0.1602 & 1.624 & 0.8186 to 3.222 \\
\hline Previous PID & $8.8(8 / 91)$ & $7.7(57 / 742)$ & 0.6794 & 1.158 & 0.5339 to 2.513 \\
\hline IUD & $0 \%$ & $1.3 \%(10 / 733)$ & 0.6121 & 2.62 & 0.1521 to 45.11 \\
\hline Mirena coil & $0 \%$ & $0.9 \%(7 / 743)$ & 1 & 1.864 & 0.1055 to 32.92 \\
\hline \multicolumn{6}{|c|}{ Clinical presentation of women with primary and recurrent EP } \\
\hline Pain $^{b}$ & $93.4(85 / 91)$ & $94.5(705 / 746)$ & 0.63 & 0.8239 & 0.3397 to 1.998 \\
\hline Bleeding ${ }^{\mathrm{b}}$ & $80.2(73 / 91)$ & $85.0(634 / 746)$ & 0.2239 & 0.7164 & 0.4118 to 1.246 \\
\hline Vomiting $^{b}$ & $7.7(7 / 91)$ & $9.7(72 / 746)$ & 0.7041 & 0.7801 & 0.3475 to 1.751 \\
\hline Diarrhoea ${ }^{b}$ & $0(0 / 91)$ & $1.9(14 / 746)$ & 0.3849 & 0.2761 & 0.01632 to 4.670 \\
\hline Shoulder tip pain ${ }^{b}$ & $4.4(4 / 91)$ & $10.5(78 / 745)$ & 0.0899 & 0.3932 & 0.1404 to 1.101 \\
\hline Syncopal attack ${ }^{b}$ & $6.6(6 / 91)$ & $11.1(83 / 745)$ & 0.2112 & 0.563 & 0.2385 to 1.329 \\
\hline 1st $\beta \mathrm{HCG}^{\mathrm{a}}$ & $3176(7350)$ & $6243(12,282)$ & 0.0099 & 3053 & 750 to 5355 \\
\hline TVS $^{\mathrm{b}}$ & $95.6(87 / 91)$ & $88.3(651 / 737)$ & 0.0323 & 0.348 & 0.1246 to 0.9724 \\
\hline TAS $^{\mathrm{b}}$ & $12.1(11 / 91)$ & $17.1(126 / 738)$ & 0.2943 & 1.497 & 0.7747 to 2.894 \\
\hline Both TVS and TAS ${ }^{b}$ & $11.0(10 / 91)$ & $12.0(88 / 735)$ & 1 & 1.102 & 0.5505 to 2.205 \\
\hline \multicolumn{6}{|l|}{ Ultrasound scan findings } \\
\hline Adnexal mass ${ }^{b}$ & $84.6(77 / 91)$ & $88.6(643 / 726)$ & 0.3007 & 1.409 & 0.7624 to 2.602 \\
\hline Gest sac ${ }^{b}$ & $24.2(22 / 91)$ & $20.3(148 / 730)$ & 0.4104 & 0.7976 & 0.4776 to 1.332 \\
\hline $\mathrm{FH}^{\mathrm{b}}$ & $12.1(11 / 91)$ & $8.9(65 / 730)$ & 0.3363 & 0.7109 & 0.3602 to 1.403 \\
\hline PoD fluid ${ }^{b}$ & $68.1(62 / 91)$ & $76.3(557 / 730)$ & 0.094 & 1.506 & 0.9385 to 2.417 \\
\hline
\end{tabular}

The comparison of the baseline characteristics, risk factors, the clinical presentations and ultrasound scan findings of women presenting with primary EP compared with recurrent EP

TVS transvaginal ultrasound scan, TAS transabdominal ultrasound scan, PID pelvic inflammatory disease, IUD intrauterine device, FH fetal heartbeat, $P O D$ pouch of douglas

${ }^{a}$ Data shown as mean (SD) and analysed by Student's t test, with mean difference and $95 \%$ confidence interval

${ }^{\text {b }}$ Data shown as \% and analysed by Fisher's exact test with Odds ratio (OR) and $95 \%$ confidence interval

follow up. The lower $\beta$ HCG level may reflect an earlier presentation of the women due to our stringent follow up protocol and counseling. Women are seen post operatively in a dedicated clinic or are telephoned in our dedicated telephone clinic and debriefed about their diagnosis and treatment. They are advised on the clinical course to follow with the next pregnancy, including getting in touch with their general practitioner as soon as the pregnancy test becomes positive. An early ultrasound scan is arranged to facilitate early diagnosis. This indicates that women are being diagnosed at an earlier stage when more tubal preservation could take place. 
Table 3 Operative findings

\begin{tabular}{|c|c|c|c|c|c|}
\hline & \multirow{2}{*}{$\begin{array}{l}\text { Recurrent ectopic } \\
\%(n)\end{array}$} & \multicolumn{4}{|c|}{ Primary ectopic } \\
\hline & & $\%(n)$ & p value & $\begin{array}{l}\text { Mean difference } \\
\text { or odds ratio }\end{array}$ & $95 \% \mathrm{Cl}$ \\
\hline Presence of haemoperitoneum ${ }^{\mathrm{a}}$ & $89.9(80 / 89)$ & $89.8(654 / 728)$ & 1 & 1.006 & $0.4847-2.087$ \\
\hline Volume of haemoperitoneum $(\mathrm{m} / \mathrm{s})^{b}$ & $249(391)$ & $592(850)$ & $<0.0001$ & 360.6 & 249.7-471.4 \\
\hline Presence of hepatic adhesions ${ }^{a}$ & $13.5(12 / 89)$ & $7.6(53 / 695)$ & 0.0666 & 1.888 & $0.9661-3.689$ \\
\hline Contralateral pathology ${ }^{a}$ & $31.1(28 / 90)$ & $9.8(74 / 755)$ & $<0.0001$ & 4.156 & $2.504-6.899$ \\
\hline Laparotomy $^{\text {a }}$ & $1.1(1 / 90)$ & $4.5(34 / 756)$ & 0.1642 & 0.2386 & $0.03225-1.765$ \\
\hline Tubal ectopic ${ }^{\mathrm{a}}$ & $97.8(89 / 91)$ & $93.3(705 / 756)$ & 0.1082 & 3.219 & $0.7702-13.46$ \\
\hline Salpingectomy ${ }^{a}$ & $57.1(52 / 91)$ & $83.3(625 / 750)$ & $<0.0001$ & 0.2667 & $0.1688-0.4214$ \\
\hline Salpingotomy ${ }^{\mathrm{a}}$ & $37.4(34 / 91)$ & $10.9(82 / 750)$ & $<0.0001$ & 4.859 & $2.998-7.875$ \\
\hline
\end{tabular}

The intra operative findings of women presenting with primary EP compared with recurrent EP

a Data shown as \% and analysed by Fisher's exact test with odds ratio (OR) and $95 \%$ confidence interval

b Data shown as mean (SD) and analysed by Student's $t$ test, with mean difference and $95 \%$ confidence interval

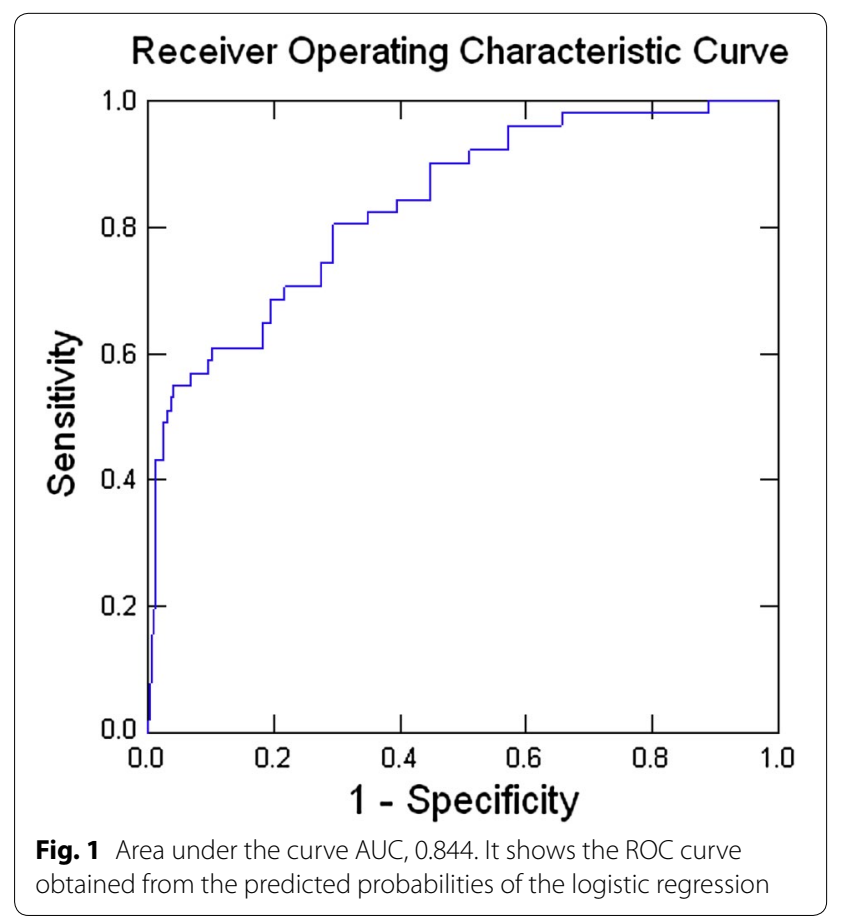

Women with recurrent EP were significantly older and a greater proportion was parous, although this did not reach statistically significance. This is consistent with the study by Butts et al. (Butts et al. 2003). In the UK, parous women are excluded from assisted conception treatment on the National Health Service and therefore everything should be done to preserve the affected tube, with the necessary surgical expertise available for management (Fertility: assessment and treatment for people with fertility problems 2013; Odejinmi et al. 2008). The ESEP study found that in women with a tubal pregnancy and
Table 4 Logistic regression analysis

\begin{tabular}{|c|c|c|c|c|c|}
\hline \multirow[t]{2}{*}{ Parameter } & \multirow[t]{2}{*}{ Odds ratio } & \multirow[t]{2}{*}{ STD error } & \multirow[t]{2}{*}{ p value } & \multicolumn{2}{|l|}{$95 \% \mathrm{Cl}$} \\
\hline & & & & Lower & Upper \\
\hline Age & 1.085 & 0.033 & 0.008 & 0.021 & 0.142 \\
\hline Gestation & 0.808 & 0.101 & 0.090 & -0.459 & 0.033 \\
\hline $\begin{array}{l}\text { Previous tubal } \\
\text { surgery }\end{array}$ & 21.133 & 9.473 & 0.000 & 2.172 & 3.929 \\
\hline $\begin{array}{l}\text { Previous pelvic } \\
\text { surgery }\end{array}$ & 2.455 & 1.008 & 0.029 & 0.093 & 1.703 \\
\hline First $\beta H C G$ & 1.000 & 0.000 & 0.058 & 0.000 & 0.000 \\
\hline
\end{tabular}

The logistic regression analysis results of the variables which were significant of univariate analysis

a healthy contralateral tube, salpingotomy does not significantly improve fertility prospects compared with salpingectomy (Mol et al. 2014). Though the limitations of the ESEP study should be taken into consideration when managing these groups of women (Odejinmi 2014).

Unsurprisingly, women with EP were significantly more likely to have contralateral tubal pathology. There has always been some debate as to what type of surgery should be performed in women with a damaged contralateral tube (The Management of Tubal Pregnancy 2004). The consensus is that women should be treated on an individual basis with informed consent. If the contralateral tube is damaged and preservation of fertility is a priority, then a salpingotomy is advised, as although this increases the chance of recurrent EP to $20 \%$, the chance of intrauterine pregnancy is $50 \%$. Extrapolation from this would mean that if women have a recurrent EP, ideally they should have a salpingotomy, which again needs additional surgical expertise. Although in the short-term a salpingotomy is more expensive, due 
to additional post-operative follow up and treatment for persistent trophoblast that may become necessary, the reduced need for assisted conception renders this treatment more cost-effective in the long-term (The Management of Tubal Pregnancy 2004; Mol et al. 1997).

The results from our logistic regression analysis of significant pre-operative characteristics emphasize that the groups are significantly different. Women with recurrent EP are more likely to be older, with a positive history of previous tubal or pelvic surgery and to present at an earlier gestation with a lower $\beta$ HCG. Therefore, it is paramount that transvaginal ultrasonography is performed by a sufficiently experienced practitioner and if surgical management is considered, then the necessary surgical expertise is available (Odejinmi et al. 2008; Brown and Doubilet 1994; Crochet et al. 2013).

It is somewhat surprising that there was no significant difference in the history of previous pelvic inflammatory disease (PID) between the two groups. One might expect that women with recurrent EP would be more likely to have a history of previous PID or any other pathology that would be directly responsible for recurrence. Our finding is consistent with some of the literature (Butts et al. 2003; Joesoef et al. 1991), but contrasts with another large study of recurrent EP, which found that a history of infectious pathology tripled the risk of recurrent EP (Skjeldestad et al. 1998). Possible explanations for this include the fact that sexually transmitted infections and PID are notoriously both under-reported and underdiagnosed (Bouyer et al. 2003). This is one of the facets of contributing risk which was not explored in our study. Our protocol for patient management during investigation and management for an EP included investigations for Chlamydia only if there was a positive history of previous/recent PID. Thus along with the majority of similar studies, actual numbers of infections could have been under reported in our study. In a previous study of EP, although less than $10 \%$ of patients gave a history of PID/ salpingitis, over $75 \%$ were found to have antibodies to $N$ gonorrhoea or C trachomatis, suggesting that infection is commonly sub-clinical (Spandorfer and Barnhart 2003). The link with sub-clinical PID and EP has been evidenced (Sweet and Gibbs 2012). And therefore, it is possible that a significant proportion of our patients had sub-clinical PID and that our results on history of previous PID are therefore unreliable. The significant impact on future reproductive health, including recurrent $\mathrm{EP}$, emphasizes the importance of establishing a history of PID and regularly testing for sexually transmitted infections, even in apparently low risk women.

When considering other established risk factors for EP, there was no statistically significant difference in risk factors between the two groups, except for previous tubal or pelvic surgery. However, there were a marginally higher proportion of smokers, women with a previous miscarriage and women with previous subfertility in the group with recurrent EP. Conversely, there were 17 women out of 758 with a primary EP who had a Mirena or copper coil in situ, compared to $0 \%$ of women with recurrent EPs (again, this did not reach statistical significance). This may represent the fact that women who have already had one EP do not wish to expose themselves to anything else which may increase their risk of ectopic implantation (Bouyer et al. 2003; Bouyer et al. 2000; Mol et al. 1995; Parashi et al. 2014). Women who want to avoid a recurrent EP and need contraception sometimes opt for an implant but this also has its drawbacks as it has been shown that although the failure rate is extremely low the risk of EP is still present (Olowu et al. 2011). The review by Rana et al. showed that surgically managed EPs are decreasing and not the actual incidence of EP (Rana et al. 2013).

Our study is limited by the fact that we have only included women who have had a surgically managed EP. Therefore, we are missing women with a recurrent EP who had conservative or medical management of their EP. At our unit, we have been collecting data on conservative or medical management since 2009-during this period there have been 13 recurrent EPs that were managed with conservative or medical management. Nevertheless, our cohort represents the largest study of recurrent EP in the literature. Expecting a recurrent EP pre operatively is of importance in planning the surgical outcome as many tubal preservation techniques are being used successfully (Watrowski 2014).

The non-tubal as well as the tubal EPs were considered together. The risk factors and etiology of non tubal and tubal implantation are not significantly different. The symptoms and signs of non tubal ectopic pregnancy do not significantly differ from those of tubal pregnancies though they tend to present later. The symptoms are similar and the only significant differences are due to the delayed diagnosis leading to accentuation of the symptoms at presentation (Alalade et al. 2015). Due to all the above factors it was felt that excluding non tubal EPs from the analysis would introduce bias.

One of the limitations of our study is that one of primary ectopic pregnancies if followed up for a further number of years could develop a recurrent ectopic. This bias in our study is limited by the fact that the numbers of primary ectopics are significantly greater than that of the recurrent ectopics. Additionally none of the primary ectopic pregnancies have developed another ectopic since July 2014. A better study design would be a cohort study, including patients who have their first ectopic pregnancy during a specific period of time and 
subsequently observed during a certain number of years in order to differentiate those patients who have recurrent ectopic pregnancies from those who do not have more ectopic pregnancies.

In conclusion, we have demonstrated that women with recurrent EP represent a unique sub-group of women with EP. They are older, more likely to present at an earlier gestation, with a lower initial $\beta$ HCG and significantly less hemoperitoneum. We found no significant difference in symptoms at presentation, or in risk factors presentexcept for a history of tubal or pelvic surgery. Unfortunately, there is a paucity of modifiable risk factors for secondary prevention of recurrent EP. The implications for future reproductive health require women with recurrent EP to be rapidly identified and managed with the appropriate level of surgical expertise.

\section{Authors' contributions}

$\mathrm{AH}$ : Project development, Data collection and management, Data analysis, Manuscript writing and editing. RO: Project development, Data collection and management, Data analysis, Manuscript writing and editing. FO: Project development, Data collection and management, Data analysis, Manuscript writing and editing. All authors read and approved the final manuscript.

\section{Competing interests}

The authors declare that they have no competing interests respect to the research, authorship, and/or publication of this article. We state that we have full control of all primary data and we agree to allow the journal to review our data if requested. All our data was stored in line with the Caldicott guidelines.

Received: 14 August 2015 Accepted: 12 February 2016

Published online: 03 March 2016

\section{References}

Alalade A, Mayers K, Abdulrahman G, Oliver R, Odejinmi F (2015) A twelve year analysis of non-tubal ectopic pregnancies: do the clinical manifestations and risk factor for these rare pregnancies differ from those of tubal pregnancies? Gynecol Surg. doi:10.1007/s10397-015-0917-1

Ankum WM, Mol BW, Van der Veen F, Bossuyt PM (1996) Risk factors for EP: a meta-analysis. Fertil Steril 65:1093-1099

Bouyer J, Rachou E, Germain E, Fernandez H, Coste J, Pouly JL, Job-Spira N (2000) Risk factors for extrauterine pregnancy in women using an intrauterine device. Fertil Steril 74(5):899-908

Bouyer J, Coste J, Shojaei T, Pouly JL, Fernandez H, Gerbaud L, JobSpira N (2003) Risk factors for EP: a comprehensive analysis based on a large case-control, population-based study in France. Am J Epidemiol 157(3):185-194

Brown DL, Doubilet PM (1994) Transvaginal sonography for diagnosing EP: positivity criteria and performance characteristics. J Ultrasound Med 13(4):259-266
Butts S, Sammel M, Hummel A, Chittams J, Barnhart K (2003) Risk factors and clinical features of recurrent EP: a case control study. Fertil Steril 80(6):1340-1344

Creanga AA, Shapiro-Mendoza CK, Bish CL, Zane S, Berg CJ, Callaghan WM (2011) Trends in ectopic pregnancy mortality in the United States: 1980-2007. Obstet Gynecol 117(4):837-843

Crochet JR, Bastian LA, Chireau MV (2013) Does this woman have an EP? The rational clinical examination systematic review. JAMA 309(16):1722-1729

Crook MA (2003) The Caldicott report and patient confidentiality. J Clin Pathol 56:426-428

Fertility: assessment and treatment for people with fertility problems. NICE Clinical Guideline 156, February 2013

Joesoef MR, Westrom L, Reynolds G, Marchbanks P, Cates W (1991) Recurrence of EP: the role of salpingitis. Am J Obstet Gynecol 165(1):46-50

Jurkovic D, Wilkinson H (2011) Diagnosis and management of EP. BMJ 10(342):3397

Mol BW, Ankum WM, Bossuyt PM, Van der Veen F (1995) Contraception and the risk of EP: a meta-analysis. Contraception 52(6):337-341

Mol BW, Hajenius PJ, Engelsbel S, Ankum WM, Hemrika DJ, van der Veen F (1997) Bossuyt PM (1997) Is conservative surgery for tubal pregnancy preferable to salpingectomy? An economic analysis. Br J Obstet Gynaecol 104(7):834-839

Mol F, van Mello NM, Strandell A, Strandell K, Jurkovic D, Ross J, Barnhart KT, Yalcinkaya TM, Verhoeve HR, Graziosi GC, Koks CA, Klinte I, Hogström L, Janssen IC, Kragt H, Hoek A, Trimbos-Kemper TC, Broekmans FJ, Willemsen WN, Ankum WM, Mol BW, van Wely M, van der Veen F, Hajenius PJ (2014) European Surgery in Ectopic Pregnancy (ESEP) study group Salpingotomy versus salpingectomy in women with tubal pregnancy (ESEP study): an open-label, multicentre, randomised controlled trial. Lancet 383(9927):1483-1489. doi:10.1016/S0140-6736(14)60123-9

Odejinmi F (2014) Oliver R (2014) Salpingotomy versus salpingectomy in women with tubal pregnancy. J Comp Eff Res 3(3):241-243

Odejinmi FO, Rizzuto MI, Macrae RE, Thakur V (2008) Changing trends in the laparoscopic management of EP in a London district general hospital: 7-years experience. J Obstet Gynaecol 28(6):614-647

Olowu O, Karunaratne J, Odejinmi F (2011) Ectopic pregnancy with implanon as a method of contraception in a woman with a previous ectopic pregnancy-case report. Eur J contracep health care 16:1-3

Parashi S, Moukhah S, Ashrafi M (2014) Main risk factors for EP: a case-control study in a sample of Iranian women. Int J Fertil Steril 8(2):147-154

Rana P, Kazmi I, Singh R, Afzal M, Al-Abbasi FA, Aseeri A, Singh R, Khan R, Anwar F (2013) Ectopic pregnancy: a review. Arch Gynecol Obstet 288(4):747-757

Saving Mothers'Lives: 2006-2008, Centre for Maternal and Child Enquiries (CMACE) (2011). Br J Obstet Gynaecol 118(1)

Schoen JA, Nowak RJ (1975) Repeat EP: a 16-year clinical survey. Obstet Gynecol 45(5):542-546

Skjeldestad FE, Hadgu A, Eriksson N (1998) Epidemiology of repeat EP: a population-based prospective cohort study. Obstet Gynecol 91:129-135

Spandorfer SD, Barnhart KT (2003) Role of previous EP in altering the presentation of suspected EP. J Reprod Med 48(3):133-136

Sweet RL, Gibbs RS (2012) Pelvic inflammatory disease_-Infectious diseases of the female genital tract. Lippincott Williams \& Wilkins, Baltimore, pp 220-245

The Management of Tubal Pregnancy, Royal College of Obstetricians and Gynaecologists Guideline No. 21, May 2004

Watrowski R (2014) Hemostatic gelatine-thrombin matrix (Floseal ${ }^{\circledR}$ ) facilitates hemostasis and organ preservation in laparoscopic treatment of tubal pregnancy. Arch Gynecol Obstet 290(3):411-415 